Introduction The effect of COVID-19 extended to undergraduate teaching, including reduced capacity around the bedside and clinical restrictions due to infection control policies. This resulted in students undertaking near-peer teaching for their colleagues. A survey of these students found that they encountered barriers to accessing, organising and delivering near-peer teaching.

Aims This quality improvement project was employed to:

- Standardise students' access to near-peer teaching opportunities

- Foster confidence in teaching and feedback skills

- Evaluate whether this scheme could add value to a clinical placement

Methods Students volunteered to become a tutor/tutee during the placement. Participants were matched based on specific criteria and preferences. A clinical teaching fellow was available for support and advice. Data was collected using online pre/post-scheme questionnaires, Likert scales, and free text questions.

Results 17 pre-scheme and post-scheme questionnaires were completed. The scheme increased tutors' confidence in delivering teaching/feedback to near-peers (100\% response). Participants felt that the near-peer teaching scheme added value to their placement and would recommend future implementation (100\% response).

Conclusion This scheme supported students to lead teaching sessions, deliver feedback, and act as mentors. Anticipated future benefits include improvement in student-patient interactions, deeper understanding of medical topics and a greater interest in pursuing educational leadership roles.

We encourage undergraduate departments to implement this scheme to support students who are enthusiastic about teaching during clinical placements. This can foster increased confidence in teaching skills, delivering feedback and improvement in leadership skills whilst adding value to the clinical placement. These valuable skills would contribute greatly towards the participants becoming well-rounded doctors.

\section{PERCEPTIONS OF MEDICAL PROFESSIONALS ON MEDICAL LEADERSHIP TRAINING IN MEDICAL SCHOOL}

${ }^{1}$ Aishah Zubaida Mughal* ${ }^{2}$ Jade Fellows, ${ }^{3}$ Haroon Ali Shah. ${ }^{1}$ University of Birmingham Medical School, Birmingham, UK; ${ }^{2}$ University Hospitals Birmingham, Birmingham, UK; ${ }^{3}$ University Hospitals of Derby and Burton, Burton-On-Trent, UK

\subsection{6/leader-2021-FMLM.51}

Background \& Aims Perceptions and attitudes amongst medical professionals, particularly medical students, surrounding medical leadership training remains little understood. There is a paucity of data surrounding the effectiveness of medical leadership teaching within medical school curriculums. This questionnaire-based study aims to explore the opinions of medical leadership training amongst those attending virtual webinars organised by a university leadership society.

Methods A questionnaire-based study was performed by a team of senior medical students on the Birmingham Medical Leadership Society (BMLS) committee at the University of Birmingham Medical School from July 2020 to January 2021. Questionnaires were offered to all attendees across 12 online webinars. Responses were recorded electronically and independently collated.
Results $88.7 \%$ of attendees were medical students, with the remaining attendees comprising of doctors (7.5\%) and other allied healthcare professionals. $32.3 \%$ of participants were interested in intercalating in medical leadership and felt opportunities to intercalate were available. $18.1 \%$ of attendees were interested in intercalating in medical leadership but felt an opportunity to intercalate was not available. $46.6 \%$ of participants strongly agreed that medical leadership should be taught at an undergraduate level, however, a third (33.1\%) disagreed that the proportion of medical leadership education delivered at medical school was adequate.

Conclusion Whilst strategies have been implemented to improve undergraduate medical leadership education, further work is required to mitigate barriers to effective leadership training. Medical schools should regularly assess the opinions of students in order to optimise medical education and ensure opportunities to explore medical leadership, such as through intercalation, are made available to all students.

Presenting author Aishah Zubaida Mughal

\section{QUALITY IMPROVEMENT PROJECT ON PAEDIATRIC ANTIBIOTIC PRESCRIPTION WRITING}

Jini Haldar. Chesterfield Royal hospital ,UK

\subsection{6/leader-2021-FMLM.52}

Aim To improve the quality of handwritten antibiotic prescriptions at the Paediatric ward of Chesterfield Royal Hospital.

Method

1. Data were collected prospectively by Microsoft Excel of all antibiotic prescriptions in May 2021 for an audit to see the baseline.

2. Quality improvement project launched with teaching session for all junior doctors with pharmacists about aim and plan with prescription MAT and with the announcement of high achievement award for the best prescriber with a chocolate box gift and a certificate. Later, for the wonderful participation, another handmade gift was announced for the best prescriber.

3. Every 2 weekly, the progress was checked and the marking of all prescriptions was done.

4. After 1st cycle there was a drop in the percentage of compliance in a few sections so the strategy was planned to remind the juniors at the hand over time, also by Whatsapp group and personal emails. Also, it was promised to arrange a meet up to give gifts to the winners.

5. 5 . After the 2 nd cycle, improvements can be seen in all fields and there was very little to improve so the strategy was to announce 2 handmade gifts to the 2 best prescribers in the last cycle who will get 10 out of 10 marks to get a star. A Star chart was implemented.

6. Finally, after a wonderful outcome each and individual who participated in the project was given one small handmade gift as a token of appreciation alongside all the winners gifts.

Results We can see a huge improvement in all sections except the section of the pharmacist that could not be improved in 3 months duration of this project for staffing issues.

Conclusion This project is hugely successful with a happy experience for all by the personal touch and by the appreciation of the efforts of Juniors in the department and also by flexibly changing strategies as per requirements. 